\title{
Apropiación social del conocimiento en cuatro grados de desarrollo empresarial en organizaciones productivas mexicanas en la frontera Sur México/ Belice. Análisis del modelo $\mathrm{OCDE}^{1}$
}

Francisco J. Güemez Ricalde ${ }^{2}$

Universidad de Quintana Roo, Chetumal, Quintana Roo. México ${ }^{3}$ fragueme@uqroo.mx

Adriana Y. Valdivieso Ortiz ${ }^{4}$

Universidad de Quintana Roo, Chetumal, Quintana Roo. México ${ }^{5}$ yarelivaldivieso@gmail.com

José L. Zapata Sánchez ${ }^{6}$

Universidad de Quintana Roo, Chetumal, Quintana Roo. México ${ }^{7}$ jzapata@gmail.com

Ma. de los Ángeles Hernández Bustos ${ }^{8}$

Colegio de Bachilleres, Chetumal, Quintana Roo. México ${ }^{9}$

mangeles37@hotmail.com

Recibido:26 de febrero de 2014

Aceptado:21 de mayo de 2014

Disponible en línea: 15 de marzo de 2015

\footnotetext{
Artículo de investigación científica y tecnológica. Proyecto financiado por el Fondo Institucional de Fomento Regional para el Desarrollo Científico, Tecnológico y de Innovación (FORDECYT), dentro del proyecto regional "Apropiación social de la ciencia, la tecnología y la innovación-Región sur Sureste del Consejo Nacional de Ciencia y Tecnología (CONACYT)”.

2 Doctorado en Ecología y Desarrollo Sustentable

3 Profesor-investigador adscrito a la División de Ciencias Socioeconómicas

4 Licenciada en Sistemas Comerciales

5 Auditora interna en la empresa ULTRAFEME

6 MC en Marketing

7 Profesor-investigador adscrito a la División de Ciencias Socioeconómicas

8 Ingeniera Agrónoma en Administración Agropecuaria

9 Profesora adscrita al COBACH II
} 


\title{
Apropiación social del conocimiento en cuatro grados de desarrollo empresarial en organizaciones productivas mexicanas en la frontera Sur México/Belice. Análisis del modelo OCDE
}

\begin{abstract}
Resumen
A partir de la inserción de México en la OCDE, el presente artículo de investigación intenta dar respuesta al tema de la innovación tecnológica para el incremento de la competitividad de las cuatro empresas más importantes del estado de Quintana Roo, México económica y socialmente,en función de su tamaño y características. La investigación formó parte de un estudio al sureste de México financiado por el FORDEC y T. El modelo vigente de la OCDE (2009-2010) con estándares sobre apropiación del conocimiento, adaptado al método de entrevista directa sirvió de análisis. Aunque los resultados mostraron un marcado atraso en las cuatro empresas analizadas en cuanto apropiación del conocimiento, los resultados arrojan diferencias étnicas y culturales de los mayas que habitan en esta región cuando se trata de empresas de tipo social. Ellos adoptan paquetes tecnológicos completos y/o modelos de mercado globalizantes; ante la imposibilidad de adoptar o apropiar socialmente el conocimiento, son dependientes de su fuerza de trabajo o bien, de sus modelos ancestrales de producción o explotación del recurso principal.
\end{abstract}

Palabras clave: apropiación del conocimiento; tamaño de empresas; educación y capacitación; innovación

\section{Social appropriation of knowledge in four degrees of business development in Mexican production organizations in the Southern Mexico / Belize border. Analysis of the Model OECD}

\begin{abstract}
From the inclusion of Mexico in the OECD, this research paper attempts to answer the question of technological innovation for increasing the competitiveness of the four largest firms in the state of Quintana Roo, Mexico economically and socially based on their size and characteristics. The research was part of a study conducted in the southeast of Mexico and it was sponsored by the FORDECyT. The current model of the OECD (2009-2010) with standards on the appropriation of knowledge, adapted to the direct interview method was used for the analysis.Although the results showed a noticeable backwardness in the four companies analyzed regarding the appropriation of knowledge, they also show ethnic and cultural differences in the Maya peoples living in this region when it comes to social businesses. They adopt complete technological packages and / or globalizing market models. When facing the impossibility of adopting or appropriating socially the knowledge, they are dependent on their workforce or their ancestral patterns of production and exploitation of the main resource.

Keywords: appropriation of knowledge; company size; education and training; innovation
\end{abstract}

\section{Apropriação social do conhecimento em quatro grados de desenvolvimento empresarial em organizações produtivas mexicanas na fronteira Sul México/Belize. Análise do modelo OCDE}

\section{Resumo}

A partir da inserção do México na $\mathrm{OCDE}$, o presente artigo de pesquisa tenta dar resposta à questão da inovação tecnológica para o incremento da competitividade das quatro empresas mais importantes do Estado de Quintana Roo, México, económica e socialmente, em função do seu tamanho e características. A pesquisa formou parte de um estudo ao sudeste do México financiado pelo FORDECyT. O modelo vigente da OCDE (2009-2010) com padrões sobre apropriação do conhecimento, adaptado ao método de entrevista direta serviu de análise. Embora os resultados mostraram um atraso marcante nas quatro empresas analisadas em quanto apropriação do conhecimento, os resultados mostram diferenças étnicas e culturais dos maias que habitam nesta região quando se tratou de empresas de tipo social. Eles adotaram pacotes tecnológicos completos e/ou modelos de mercado globalizantes; perante à impossibilidade de adotar ou apropriar socialmente o conhecimento, são dependentes da sus forca de trabalho ou bem, dos modelos ancestrais de produção ou exploração do recurso principal.

Palavras-chave: apropriação do conhecimento; tamanho de empresas; ensino e capacitação; inovação 


\section{Introducción}

Sin ser Quintana Roo un estado caracterizado por el desarrollo industrial, ya que tiene vocación eminentemente turística, tiene empresas relacionadas con la explotación y un cierto nivel de transformación de productos a nivel agregado, las más grandes -casi siempre relacionadas con los consorcios o las organizaciones de tipo social- son las que técnicamente han encontrado mayor nivel de innovación y desarrollo tecnológico al estar vinculadas a mercados internacionales durante algunos años (COQCyT, 2004).

Sin duda en Quintana Roo también existen empresas de tipo familiar constituidas como microempresas que después de algunos años están siendo exitosas, mientras que otras que tienen el mismo tiempo de existencia no poseen el mismo curso que las anteriores o han tenido que cerrar, de esta manera surge una pregunta importante: ¿Qué están haciendo en materia de nuevo conocimiento las organizaciones productivas respecto al uso de los recursos para aplicar tecnologias e innovaciones que las induzcan a mejorar su condición?

En el mundo actual globalizado, se han realizado estudios internacionales (OCDE, 2009) que sugieren que entre más conocimiento, innovación y tecnología adquirida por una empresa, mayor es su desarrollo y las oportunidades de obtener éxito en el mercado correspondiente. Sin embargo, para el caso de nuestro estado, poco o nada se sabe en esta materia. Por ello se ha decidido analizar qué tipo y nivel de conocimiento utilizan las empresas quintanarroenses; si lo que está contribuyendo para el mejoramiento de dichas empresas emerge dei deas y/o factores internos (experiencias) o bien, hacen uso de recursos externos como la capacitación impartida por otras instituciones o a través de campañas del gobierno, la adquisición de nuevas tecnologías, recomendaciones o estudios realizados por organismos dedicados a la investigación e innovación.

De esta manera se darán a conocer las brechas que existen hoy día en la sociedad productiva de Quintana Roo, es decir, se busca determinar las oportunidades que las empresas (organizaciones productivas) 
no están aprovechando para mejorar su condición, o bien conocer los factores externos e internos que determinan estas brechas.

\section{Objetivos}

\section{Objetivo general}

Analizar las condiciones de apropiación social del conocimiento en las organizaciones empresariales del estado Quintana Roo, determinando los mecanismos de acción y el estado del uso, de dónde surgen, qué factores afectan la aplicación de los conocimientos y cómo afectan a sus resultados.

\section{Objetivos especificos}

- Determinar el estado y las condiciones de la aplicación del conocimiento en las empresas de tipo social y de propiedad privada en Quintana Roo.

- Identificar las formas de organización de las empresas en estudio para determinar el alcance en el aprovechamiento del uso y sistematización de nuevo conocimiento, estableciendo la forma específica de fuentes de información que les permita acceder a nuevas ideas, tecnologías y/o conocimientos.

- Identificar las oportunidades en las cuales se concentra el uso y la innovación del conocimiento, así como aquellas que están dejando pasar por no tener una gestión y sistematización interna en la aplicación del conocimiento. 


\section{Metodologia}

Se aplicó el método descriptivo con información obtenida mediante encuesta a profundidad. Las cuatro empresas que se eligieron para este estudio fueron seleccionadas de manera deliberada en función de su tamaño, diversidad sectorial en relación con la producción e importancia económica en la región.

Se analizó su forma de obtener información y cómo se generan nuevas ideas y procesos en la empresa. Esta información nos lleva en parte a medir la capacidad que tiene la empresa para ser exitosa y cómo ayuda el entorno. El estudio se llevó a cabo en empresas productivas del estado de Quintana Roo, organizaciones que por ser de distintos tamaños, captan nuestra atención y el deseo de conocer sus relaciones y/o vínculos de información.

El estudio realizado constituye un elemento de evaluación que aporta parámetros de éxito que pueden utilizarse a futuro en otras organizaciones, por lo que se establece como un primer acercamiento al tema de la apropiación del conocimiento en la entidad, al no existir estudios previos. De manera adicional, también se brinda información relativa a algunos de los problemas más comunes por los que pasan este tipo de empresas en la zona y cómo se enfrentan y superan regularmente. La confidencialidad de la información proporcionada por cada empresa es la mayor certeza de la información.

En general, a lo largo de la investigación nos preguntamos por los resultados que se han obtenido en proyectos para que cada empresa se adapte y permanezca en el mercado y en su caso, logre un mayor desarrollo. Analizamos sus vínculos con organizaciones educativas y de investigación, con su entorno y el porqué deciden buscar en otros lugares. También interrogamos y analizamos el proceso de integración y capacitación del personal, ambos importantes para el crecimiento y la aplicación de innovación tecnológica en el proceso productivo. Finalmente, incorporamos al análisis el efecto de la globalización como un posible factor detonante de la apropiación social del conocimiento de las empresas. 
El instrumento de recolección de información empleado en los cuatro casos es una adaptación al empleado por la organización para la Cooperación y el Desarrollo Económico (OCDE, 2005) que ha elaborado un modelo para la recolección de datos sobre la innovación y que asi mismo facilita una guía para interpretar dichos datos. Este manual ha sido aprobado por el Comité de Política Científica y Tecnológica de la OCDE, (CSTP).

\section{Preguntas de investigación}

Las preguntas de investigación que se intentaron responder y que guiaron la investigación fueron las siguientes:

- ¿Qué están haciendo en materia de nuevo conocimiento las organizaciones productivas del centro-sur de Quintana Roo?

- ¿Se han apropiado de mayor conocimiento con respecto a la organización o recursos, para aplicar tecnologías e innovaciones que las induzcan a mejorar su condición?

- ¿Destinan recursos para aplicar tecnologías e innovaciones?

- ¿Hay algún resultado medible en relación a este nuevo conocimiento?

\section{Limitaciones}

Esta investigación se realizó en un periodo aproximado de diez meses en el estado de Quintana Roo. Contó con escasos recursos aportados por el Consejo Quintanarroense de Ciencia y Tecnología mediante el proyecto FORDECYT. Las organizaciones objeto de estudio fueron:

- Ingenio Azucarero San Rafael de Pucté

- Hidroponia Maya S.A. de C.V. 
- Consorcio Corporativo de Productores y Exportadores en Foresteria, S.C. de R.L.

- Sociedad comercial Orca S.A.de C.V.

\section{Hipótesis}

La hipótesis principal que da origen a la búsqueda de respuestas es: La gestión del conocimiento en las organizaciones productivas del estado de Quintana Roo no ha contribuido en forma eficiente a mejorar su grado de desarrollo tecnológico y de innovación, este grado de desarrollo en la innovación y aplicación del conocimiento se asocia comúnmente en el entorno, a la solución de problemáticas en tiempo y forma que afectan la vida diaria de las empresas por carecer de una cultura de apropiación y gestión del conocimiento asociada al mal diseño o a la falta de políticas empresariales de innovación. Por lo anterior la apropiación social del conocimiento en las empresas productivas del estado de Quintana Roo se caracteriza por su escaso desarrollo.

\section{Fundamentación teórica de la investigación}

El marco conceptual y de referencia obliga a entender y asumir posiciones respecto a las diversas formas de analizar y explicar la gestión del conocimiento, por lo que adoptaremos una mezcla de las definiciones de los siguientes autores: Goñi (2010), Hamel (1990), Chaparro, (2003) y Leonard Barton (1995). El conocimiento no es un bien escaso y se ha convertido en un atractivo estratégico para las organizaciones de hoy en dia, por lo que tiende a adaptarse su aplicación en cada caso.

Al leer nos damos cuenta que coincidimos con la definición de Goñi (2010) debido a que hemos estipulado que la innovación es una característica principal para competir en el mercado, de la misma forma coincidimos con la definición de Porter (1980), pues este es para nosotros un proceso que resulta de las capacidades de aprendizaje 
tecnológico de las empresas, ya que mientras obtengamos más conocimientos, es mucho más fácil poder innovar.

De acuerdo con estos dos conceptos, optamos por adquirir el segundo, porque se adecua más a la forma de análisis de este trabajo, debido a que gestionar para nosotros, es ese conjunto de trámites que debemos llevar a cabo para resolver un asunto, buscando la actualización y vinculación con otras instituciones que nos puedan ofrecer nuevos procesos para la dirección de una empresa o negocio.

La gestión del conocimiento es un concepto que para algunos es nuevo, mientras que para otros ya es algo conocido (Drucker 1992). Para esta investigación es importante adoptarlo debido a que se necesita conocer realmente qué es gestión del conocimiento para la aplicación de este. Debido a las características de este trabajo, se opta por aceptar la definición del inglés Knowledge Management( Hamel, 1990), que es la más completa y descriptiva para entender el concepto y que coincide con el modelo de Oslo (2005) OCDE, en retomar cuatro componentes principales para medir la apropiación del conocimiento en organizaciones empresariales:

1. La tecnología es el resultado de la gestión del conocimiento, por lo tanto son herramientas útiles que se aprovechan para agilizar los procesos de las organizaciones empresariales, por lo tanto para esta investigación el concepto más propio es el ofrecido por el diccionario de la Real Academia Española, debido a que tiene un concepto amplio de tecnología que nos permite indagar y no estancarnos en un lineamiento ya que abarca gran parte de las características que se pretende dar a comprender (Acevedo, 2003).

2. La vinculación es una parte importante de este trabajo debido a que así es como se hacen las relaciones entre organizaciones empresariales, en este punto el concepto más aceptable y que cubre las características y necesidades de este trabajo es el del contador Víctor Manuel Alcántar Enríquez (2006).

3. La capacitación es uno de los procedimientos que le sirve a una organización a poner al día a sus empleados y así seguir actualizados 
para que la empresa siga creciendo, de hecho es uno de los puntos que se estaban buscando en la encuesta que se realizó a cada tipo de organización.

4. El manejo de datos, la información estadística, la búsqueda de nueva información en materia de innovación tecnológica, el uso de redes, la asistencia a foros y la suscripción a revistas especializadas, entre otras fuentes de datos representan, no solo interés sino una forma de actualización y por tanto de apropiación del conocimiento (Kuan-Tsae, Yang y Wang, 1999).

La premisa fundamental de análisis teórico se basa en la aceptación de que la apropiación se ve desde la manera de tomar algo y quedárselo, esto es lo que vamos a investigar de las empresas ya mencionadas porque deseamos ver qué tan propio es el conocimiento en su desarrollo empresarial y cómo se maneja en su beneficio con el fin de darle un fin a la apropiación según lo que proponen Davenport, T. H. y Prusak, L., (2001).

El modelo de gestión del conocimiento que se propone en el estudio parte del reconocimiento de la necesidad de la organización de mejorar los recursos corporativos de información, tecnológicos, humanos y financieros. Se centra en la gestión de información para obtener y ordenar el conocimiento organizacional y emplea para ello proyectos en los procesos de diagnóstico, diseño, implementación y evaluación. Jerarquiza el uso de la herramienta portal para compartir y utilizar el conocimiento en el desarrollo cualitativo de la organización y para evaluar la expresión de la gestión del conocimiento organizacional ${ }^{10}$.

En el estado Quintana Roo, tenemos fuentes de conocimiento como la Universidad de Quintana Roo, el Instituto Tecnológico de Chetumal, el Instituto Tecnológico de la Zona Maya, el INIFAB, el Colegio de la Frontera Sur, entre otros, los cuales apoyan a la distribución del conocimiento.

\footnotetext{
${ }_{10}$ Véase: Soto B. Maria A. y Barrios F. Norma M. Gestión del conocimiento. Parte II. Modelo de gestión por procesos.
} 
Desde tiempo atrás, la Secretaría de Desarrollo Económico del Gobierno del estado (SEDECO), realiza diversos programas y proyectos para apoyar a los empresarios de Quintana Roo, entre los más conocidos está el de "Gestión de apoyo a empresarios para participar en ferias y exposiciones nacionales e internacionales"11, que consiste en fomentar la participación de los empresarios locales en eventos nacionales e internacionales, para la búsqueda de nichos de mercado e incorporarlos al encadenamiento productivo donde necesariamente se requiere incorporar nuevo conocimiento. Así como este, también se manejan otros proyectos para brindar a las empresas quintanarroenses información, capacitación y aplicación en sistemas de calidad y mejora de procesos, como el proyecto "Comité Nacional de Productividad e Innovacion Tecnologica, A.C. (COMPITE)"12, que permite desarrollar las alternativas antes mencionadas. Los anteriores son dos de muchos otros programas que maneja la Secretaria de Desarrollo Económico. Así mismo encontramos al COQCYT que apoya a la formación de investigadores en Centros e Instituciones de Educación Superior y que es esencial para impulsar el desarrollo Científico y Tecnológico de la entidad.

\section{Resultados}

Con base en el seguimiento de indicadores y análisis de los factores determinantes para el análisis del grado de apropiación del conocimiento señalados por la OCDE en el Manual de Oslo (2005), en sus páginas desde la once hasta la dieciséis, se presentan los siguientes resultados:

El conocimiento en el estado de Quintana Roo se genera y difunde a través de instituciones como el Consejo Quintanarroense de Ciencia y Tecnología (COQCYT), la Secretaria de Desarrollo Económico (SEDECO), las instituciones educativas a nivel superior -entre ellas la Universidad de Quintana Roo, el Instituto Tecnológico de Chetumal,

\footnotetext{
${ }^{11}$ La secretaria de desarrollo económico, http://tramites.scontraloriaqroo.gob.mx/tramite_web/ ver_servicio.php?id_servicio $=807$

12 http://tramites.scontraloriaqroo.gob.mx/tramite_web/ver_servicio.php?id_servicio=1202
} 
el Instituto Tecnológico Agropecuario, entre otras- que elaboran proyectos para desarrollar herramientas que faciliten la mejora de generación y vinculación de conocimientos tecnológicos y administrativos para las empresas del estado.

También podemos concluir que existen empresas de la región con muy altos niveles de innovación tecnológica, aunque tecnologia a final de cuentas importada y con un alto costo de implementación, como el caso de Hidroponia Maya en el centro del estado. Según la encuesta, su mayor fuente de adquisición de conocimiento ha sido por parte de la asistencia a foros y congresos de actualización, del mismo modo esta misma empresa genera información de sus proveedores y clientes potenciales al grado de desarrollar nuevos productos sobre demanda. Sin embargo, aún no se crea el hábito de tener un departamento de vinculación.

Hidroponia Maya ha trabajado en el cumplimiento estricto de diferentes normas de inocuidad alimentaria, salubridad entre otras, y ha obtenido certificaciones que le permiten comercializar en México y en el extranjero, debido a que es una empresa que exporta productos de consumo para el mercado internacional, aunque sean estos, mercados monopolizados casi siempre por los mismos que proporcionan la tecnología.

La tecnología de la empresa Hidroponia Maya es de origen italiano, según se mencionó en la encuesta. Comprende lo que es la maquinaria (seleccionador de tamaño de los pepinos), las máquinas dosificadoras de agua (para los cultivos en el invernadero) y el software. Cada una de estas se actualiza cada determinado tiempo ${ }^{13}$. Para la adquisición de lo anterior, Hidroponia Maya consultó a especialistas para adquirir la tecnologia que le otorgara la mayor eficiencia y que fuese la correcta para el tipo de trabajo que realiza la empresa en la región.

En cuanto a la capacitación, esta empresa realiza capacitaciones anualmente y ya tiene establecido un calendario de actividades para abastecer a sus trabajadores de nueva información y actualizarlos en

${ }^{13}$ Afirmación del Ing. Sergio Antonio Enriquez Reyes, Hidroponia Maya. 
lo relativo a lo que hacen en la planta, es decir, no se capacita en un solo tema, sino que la capacitación se da en función del área. Casi siempre es impartida por el proveedor de la tecnología para adecuar el manejo del producto.

Otra empresa sobresaliente en Quintana Roo es el ingenio azucarero San Rafael de Pucté que a lo largo de su trayectoria, se ha mantenido por años como uno de los ingenios en el país que produce la tercera parte del azúcar nacional. Por lo mismo, exporta a diferentes países y es considerado en la región como uno de los mayores generadores de empleo, debido a que da trabajo en temporada alta (durante la zafra) a 500 empleados directos y unos 3,500 indirectos. El ingenio pertenece a la asociación Beta San Miguel, propietaria de otros ingenios a lo largo del país (México), por lo tanto se dice que intercambia información con el resto. Esto le permite obtener nuevos conocimientos. Capta nuevas formas de tecnología comparando lo que se hace en otros ingenios dentro y fuera del país, como el ingenio Pantaleón de Guatemala, esto representa una asociación de profesionales e instituciones.

El ingenio Rafael de Pucté obtiene nuevos conocimientos y tendencias tecnológicas por medio de asistencia a congresos, seminarios y/o reuniones que se llevan a cabo por parte de asociaciones internacionales. De igual manera, el ingenio San Rafael genera y maneja la propia experiencia a través de los años para desarrollar nuevos conocimientos con respecto a la mejora de procesos de producción. Las sugerencias que les ofrecen los ingenieros como modificar ciertas temperaturas, aflojar o apretar una pieza para provocar mayor alcance, se implementan en la práctica a manera de pruebas antes de aplicarla gran escala. El ingenio azucarero tiene asociación con escuelas de nivel superior como lo es el Instituto Tecnológico de la zona maya, que le provee personal para emplear en la planta. En cuanto a la captación del personal, el ingenio San Rafael de Pucté tiene establecidos programas internos que ofrece anualmente.

Del mismo modo, el Consorcio Chiclero es una de las empresas con larga trayectoria en la entidad. Sin embargo, apenas tres años atrás ha incorporado innovaciones de tipo comercial gracias a la adquisición de nueva tecnología e información acerca de cómo elaborar 
goma orgánica, un nuevo producto: el Chicle Orgánico con saborizante, con gran aceptación en el mercado europeo.

Esta empresa adquirió el nuevo conocimiento de investigadores extranjeros, en ferias y congresos fuera del país (Alemania, entre otros). Del mismo modo, esta empresa tiene asociaciones profesionales con la Unión Nacional de Organizaciones de Forestería Comunal (UNOFOC) y la Forest Stewardship Council (FSC), que le han permitido rodearse de otras empresas, adquirir conocimientos de ellas y hasta obtener certificaciones (en el caso de la FSC). Han adoptado diferentes técnicas como resultado de la apropiación del conocimiento, lo que les ha permitido seguir creciendo e innovando. En materia de tecnología, el Consorcio Chiclero ha diseñado su propia maquinaria y con ayuda de ingenieros (fuera de la empresa), han creado una maquinaria única en el mercado. En cuanto a la capacitación de sus trabajadores, estos reciben capacitación con base en las necesidades que se presentan y no precisamente en correspondencia a un programa establecido como en otras empresas.

Por otra parte, una de las empresas familiares que se ha mantenido por muchos años en la región es la purificadora de agua La Orca, que a sus 32 años de abrir ha estado avanzando poco a poco como una empresa netamente de la región. La Orca en nuestro estudio, representa la empresa más pequeña y la única de tipo familiar. Recolecta principalmente información relativa a tecnología y conocimientos por medio de revistas científicas y de comercio y/o publicaciones técnicas, que son de mayor facilidad de manejo. Del mismo modo utiliza asociaciones de profesionales de la industria en la que se encuentra y su propietario asiste a conferencias, ferias y congresos, que son una fuente cercana y práctica de ver lo que se ha desarrollado en la industria. También capta información por medio de instituciones gubernamentales para capacitar a su personal, aunque esto último se da en función a su afiliación a la Cámara de Comercio y no propiamente en atención a necesidades específicas. 


\section{Apropiación del conocimiento en la innovación de productos}

De acuerdo al estudio realizado en campo, tres de las cuatro empresas analizadas dijeron incorporar innovaciones en sus productos, teniendo en común la práctica de innovar sus productos cada tres años. El origen del conocimiento es netamente interno en todos los casos, con ideas propias y sin apego a los estándares referentes al modelo de OCDE (Tabla 1 y Gráfico 1).

Tabla 1. Origen de la innovación de los productos.

\begin{tabular}{|c|c|c|c|}
\hline & $\begin{array}{l}\text { Origen de la } \\
\text { innovación }\end{array}$ & \multirow{2}{*}{ Total } \\
\hline & & Interno & \\
\hline \multirow[t]{4}{*}{ Empresa } & Hidroponia Maya & 1 & 1 \\
\hline & Consorcio Chiclero & 1 & 1 \\
\hline & Ingenio San Rafael de Pucté & 1 & 1 \\
\hline & Agua purificada la Orca & 1 & 1 \\
\hline Total & & 4 & 4 \\
\hline
\end{tabular}

Fuente: entrevista personal (2011)

Gráfico 1. Tipo y origen de información recopilada por la empresa con fines de innovación.

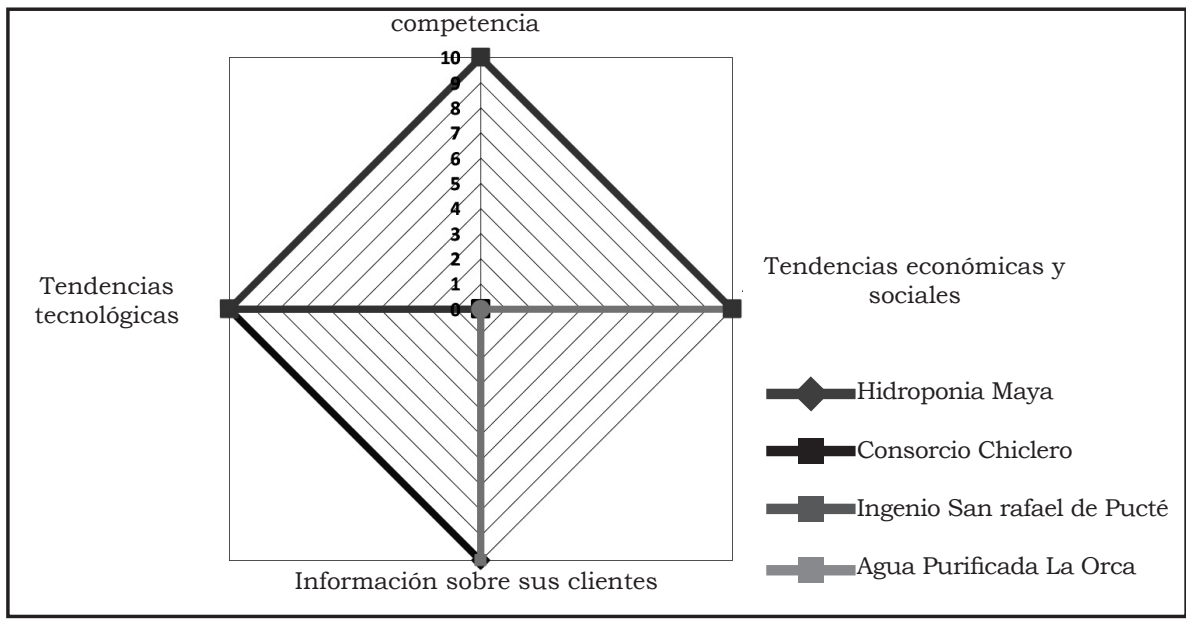

Fuente: elaborada con datos de entrevistas personales (2011) 


\section{Apropiación del conocimiento en materia de capacitación}

Las empresas más grandes cuentan con un programa anual de capacitación que se diseña en función de sus necesidades y está orientada al manejo de nueva maquinaria, o bien a la operación para la producción del nuevo producto. En otro caso, la capacitación es solo con base a alguna necesidad concreta y en la microempresa la capacitación se adapta al programa que tiene la Secretaría de Economía. En todas las empresas estudiadas la capacitación es de origen interno, sin un plan anual ni con enfoque de competitividad acorde al modelo de Oslo.

Tabla 2. Espaciamiento temporal de la incorporación de innovación en la capacitación.

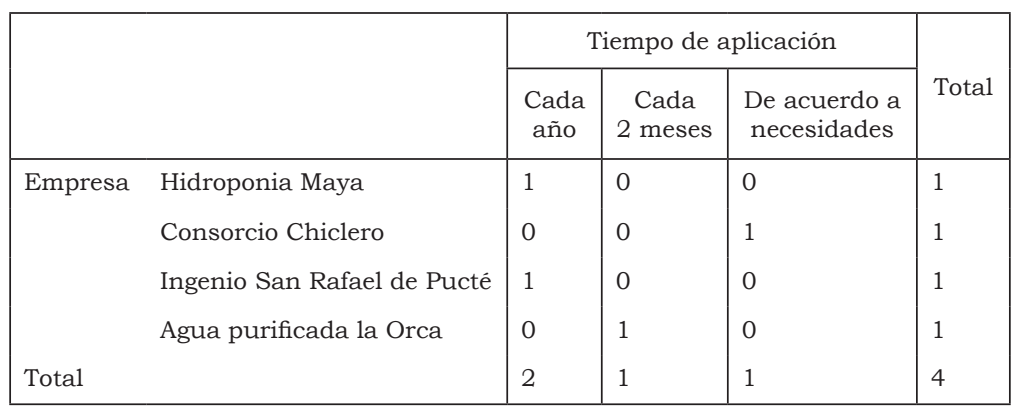

Fuente: entrevista personal (2011)

\section{Autoevaluación de las empresas respecto a la apropiación del conocimiento}

La autoevaluación arroja calificaciones desde la perspectiva del dueño de la empresa o gerente en materia de apropiación del conocimiento. Esto de alguna manera refleja su baja perspectiva de innovar y la importancia que esta tiene en el desarrollo de sus empresas. Se analizan los principales rubros considerados en el instrumento de la OCDE (2009-2010) en el gráfico 2. 
Gráfico 2. Cómo se califica del 0 al 10 respecto a la apropiación de nuevo conocimiento.

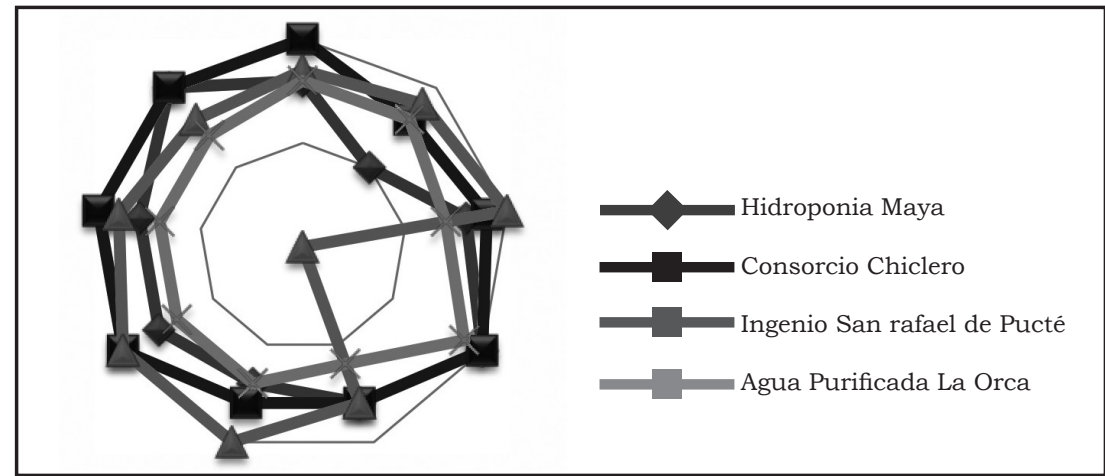

Fuente: Elaborada con base a datos de entrevistas (2011)

\section{Vinculación de las empresas con instituciones que aplican el conocimiento en la región}

El origen institucional de procedencia en materia de innovación tecnológica que en su caso solicita cada una de las empresas, es ajeno a los centros de investigación y educación superior. La excelencia ante todo, es el principal factor determinante para confiar en un programa de vinculación con fines de capacitar a su personal, es por ello que algunas empresas como Hidroponia Maya prefiere emplear consultores y asesores externos para la aplicación de las capacitaciones.

Gráfico 3. En qué porcentaje recibe la capacitación cada uno de actores.

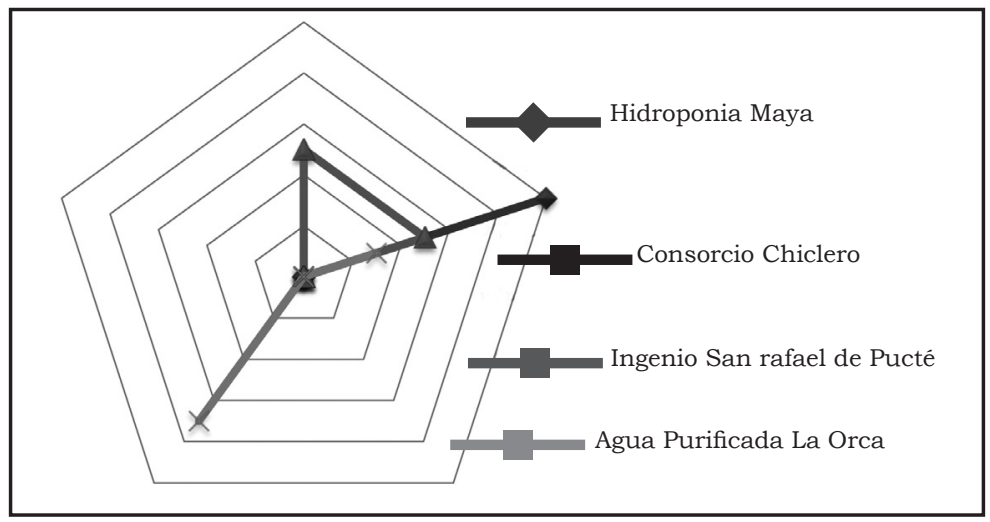

Fuente: Elaborada con base a datos de entrevistas (2011) 
Gráfico 4. Manejo de datos, Transferencia de tecnología y de información científico-técnica.

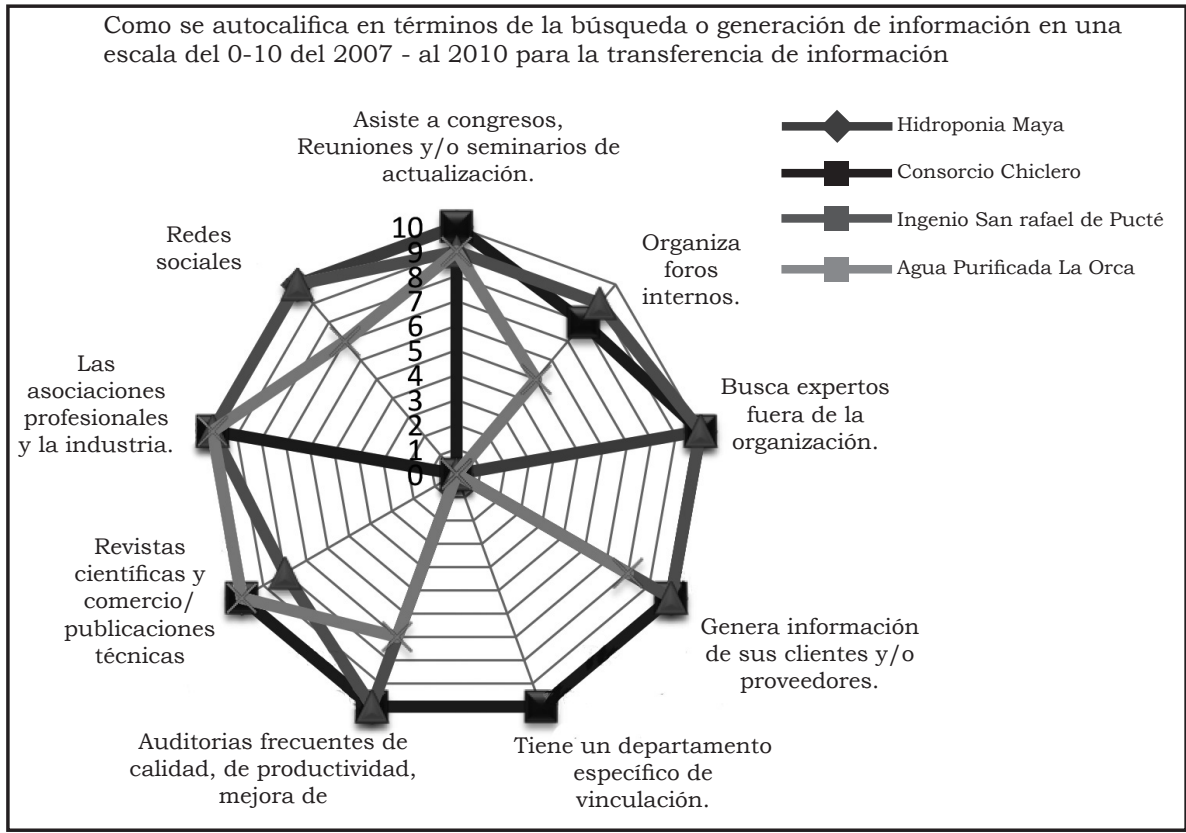

Fuente: elaborado con base a datos de entrevistas (2011)

\section{Conclusiones}

Las cuatro empresas analizadas muestran áreas de oportunidad que no son debidamente atendidas con base a un plan para el incremento de la competitividad basado en el modelo OCDE, por lo que se concluye que no utilizan plenamente el conocimiento como su principal fuente de desarrollo. La incorporación de la tecnología más actual, la capacitación, la vinculación y el análisis de datos e información señalados en el modelo de Oslo de la OCDE (2005) para medir la apropiación social del conocimiento en empresas, son nulos e incipientemente alineados a los estándares requeridos en las cuatro empresas en estudio, según nuestros resultados de la investigación.

La vinculación con los centros de investigación y desarrollo de nuevas tecnologías no existen en ningún caso (Gráfico 3). La incorporación 
de nuevas tecnologías responde más bien a necesidades específicas de la tecnología que demanda el mercado, casi siempre monopólico específico al que se vende, cito el caso de la totalidad de los niveles o tamaños de empresa en estudio: Hidroponia Maya. Consorcio Chiclero y Caña de Azúcar y empresa familiar Orca.

La capacitación se caracteriza para la adopción de la tecnología importada o el acondicionamiento a las nuevas características del producto para el mercado coincidiendo con los intereses del inversor de la tecnología y no de la región, esto no corresponde a un proceso de adopción, es más bien un entrenamiento para el trabajo o la máquina.

La capacitación, casi siempre impartida por consultores o asesores subcontratados por programas de gobierno, correspondió a programas gubernamentales casi siempre con la intención de cumplir las metas gubernamentales. La capacitación por tanto se destina a promover mayor conocimiento en las áreas administrativas de las empresas con relación a temas organizacionales, al grado de saturar el tema y la voluntad de participar, según entrevistas a profundidad.

El manejo de datos o información estadística sea de mercado, de producción o competitividad empresarial, se remite a métodos manuales mediante libreta cuando este existe, aunque se dijo que se consulta en internet o se asiste a foros para conocer posibles tendencias en otros sitios. Se señala mayor interés en los datos referentes a estadísticas de comportamiento de mercado. Ninguna de las cuatro empresas, sin importar el tamaño, dijo estar suscrito a newsletters o publicaciones científicas especializadas.

Aplicaciones mercadológicas, de manejo de la cadena de distribución o de aplicaciones o anexión al concepto de responsabilidad social, adecuaciones tecnológicas de o para la producción,s on nulas. La respuesta a las necesidades tecnológicas se dan sobre la marcha cuando se presenta el problema, como en el caso de nuevas enfermedades detectadas en la producción de caña o la baja en la productividad y el rendimiento por hectárea, o bien, el uso de la nueva tecnología de ósmosis inversas en combinación con el suo de ozono para la purificación del agua en el caso de la empresa familiar Orca. 
Los resultados finales del estudio nos permitieron determinar también que, pese a que las empresas grandes tienen mayor número de necesidades de apropiación del conocimiento y a pesar de su mayor capacidad financiera, el interés por la apropiación del conocimiento y la vinculación con los centros regionales de investigación es muy bajo o nulo, por lo que el tamaño de la empresa no representa diferencias significativas. Del mismo modo se puede apreciar que en el estado de Quintana Roo hay un déficit de instituciones educativas y sobretodo de centros de investigación y desarrollo de nuevas tecnologías aplicadas a la producción, escasamente se reportaban nueve en todo el estado en 1995,incluyendo instituciones privadas dedicadas a la docencia.

El Consejo Quintanarroense de Ciencia y Tecnología ofrece apoyo a las empresas, del mismo modo la Secretaría de Desarrollo Económico busca ofrecer, mediante programas, capacitación a las empresas, sin embargo los resultados muestran que no existen cambios en la competitividad de las empresas estudiadas.

Las empresas necesitan abrirse a nuevas alternativas de adquisición de conocimientos tecnológicos y administrativos. El nivel que se observa actualmente en materia de innovación tecnológica es muy bajo, pese a la percepción que tienen de su empresa las personas que las dirigen (Gráfico 2). Áreas tan importantes y no tan costosas como la práctica mercadológica, el uso de tecnologías y la comunicación a través de redes sociales son poco o nada utilizadas en las empresas estudiadas.

Figura 1. Envasado a mano y empaquetamiento de chicle natural para exportación.

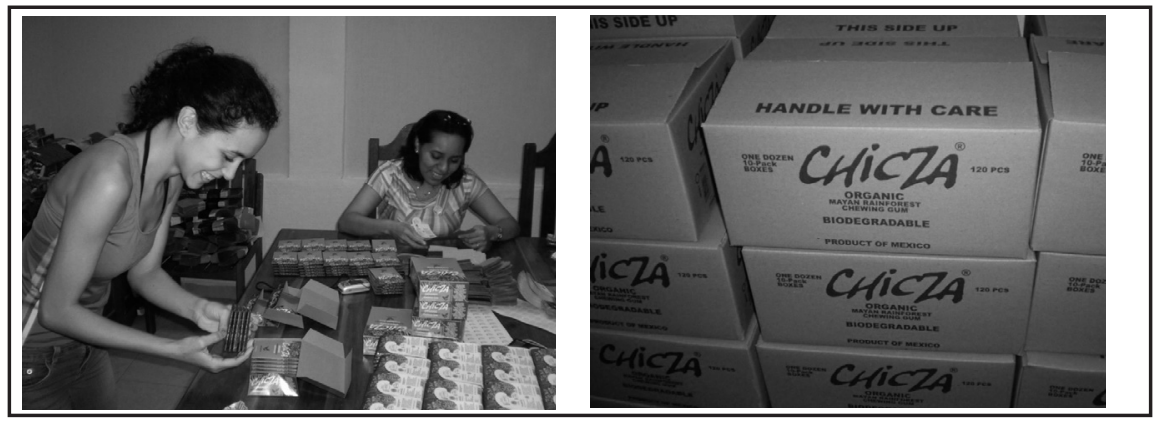

Fuente: fotografia propia (2011) 
Para alcanzar el modelo de apropiación social del conocimiento que exige la incorporación al modelo globalizado de la OCDE, es necesario que se vean reflejados a través de un modelo de generación de conocimiento mediante dos espirales, de acuerdo al modelo japonés de generación del conocimiento de Nonaka y Takeuchi (1995). Este modelo plantea un proceso de interacción entre el conocimiento tácito y explícito que tiene naturaleza dinámica y continua.

La gestión del conocimiento debe corresponder a un modelo que permita crear, compartir y gestionar conocimientos mediante la creación de una red de diferentes instituciones que en primera instancia, permita la interacción, debate y creación de productos entre responsables de centros educativos, estudiosos y gestores de la administración pública que se conocen aunque aún no comparten el interés por impulsar el sector empresarial.

La red puede tener como principales objetivos el impulso de las actividades principales señaladas en el mismo modelo OCDE para lograr la apropiación del conocimiento en las empresas: la innovación tecnológica (comercialización, mercadotecnia, comercialización, desarrollo e innovación de nuevos productos y tecnologías), el manejo, recopilación y transferencia de datos e información, la capacitación y la vinculación con los centros de investigación y desarrollo de nuevas tecnologías y centros educativos. También es necesario incorporar el apoyo tangencial financiero de los programas gubernamentales para el logro de los objetivos señalados.

Según el nuevo informe de la Organización para la Cooperación y el Desarrollo Económico (OCDE 2009), las universidades pueden jugar un papel mucho más importante en el desarrollo económico regional, social y cultural de sus regiones. El informe que se da establece que el compromiso regional que asuman las universidades será beneficioso tanto para el desarrollo local como para ellas mismas en tanto instituciones. 


\section{Referencias}

Acevedo, P. E. (2003). Innovación tecnológica, economía y sociedad: una reflexión necesaria para CTS. Colombia: Cátedra CTS+I en Colombia de la OEI.

Alcántar Enríquez, V. M. (2006). Vinculación y posicionamiento de la Universidad Autónoma de Baja California con su etorno social y productiv. Tijuana: ANUIESUniversidad Autónoma de Baja California.

Barton, L. (1995). Knowledge Management: The Cutting Edge for the 21 st Century. USA: Harvard Bussines School.

Chaparro F. (2003). Apropiación social del conocimiento, aprendizaje y capital. Colombia: Corporación Colombia Digital.

COQCyT (2004). Diagnóstico de la ciencia y tecnología en Quintana Roo. México: Impresos del Gobierno del estado de Quintana Roo.

Davenport, T. H. y Prusak, L., (2001). Conocimiento en acción. Cómo las organizaciones manejan lo que saben. Buenos Aires: Prince Hall.

Drucker P. (1992, septiembre-octubre). La nueva sociedad de organizaciones. Harvard Bussines Review, 1-11

Goñi, Z. J. (2010). Talento, tecnología y tiempo. Los pilares de un progreso consciente para elegir un futuro., Barcelona, España: Ediciones Diaz de Santos.

Hamel, P. (1990). The core competence of the corporation. USA: Harvard Bussines School.

Kuan-Tsae, H, Yang, W. y Wang, R. (1999). Calidad de la información y gestión del conocimiento: Madrid, España: Editorial AENOR.

Nonaka, I y Takeuchi, H (1995). The Knowledge creating company. Nueva York, USA: Oxford University press.

OCDE. (2005). Manual de Oslo. Guía para la recogida e interpretación de datos sobre innovación. Oslo: Comisión Europea 3a ed. 
OCDE. (2009). La politica regional de innovación de la próxima generación: cómo combinar los enfoques del impulso por la ciencia y por el usuario en los sistemas regionales de innovación. (Versión Final: 2009). Oslo: La Cuarta Encuesta de Innovación de la Comunidad Europea.

Porter, M. (1980). Estrategy Competitive. Harvard: USA.

\section{Cómo citar este artículo}

Güemez-Ricalde, F. J., Valdivieso-Ortiz, J. L. y Hernández-Bustos, Ma. de los A. (2015). Apropiación social del conocimiento en cuatro grados de desarrollo empresarial en organizaciones productivas mexicanas enla frontera Sur México/ Belice. Análisis del modelo OCDE. Universitas Humanistica, 80, 325-346. http:/ /dx.doi.org/10.11144/Javeriana.UH80.ascc 